\title{
QUEEN'S
UNIVERSITY
BELFAST
}

\section{Improving signal reliability in outdoor body-to-body communications using front and back positioned antenna diversity}

Cotton, S. L., Scanlon, W. G., \& McKernan, A. (2012). Improving signal reliability in outdoor body-to-body communications using front and back positioned antenna diversity. In 2012 6th European Conference on Antennas and Propagation (EUCAP) (pp. 3393-3396) https://doi.org/10.1109/EuCAP.2012.6206649

Published in:

2012 6th European Conference on Antennas and Propagation (EUCAP)

Document Version:

Peer reviewed version

Queen's University Belfast - Research Portal:

Link to publication record in Queen's University Belfast Research Portal

Publisher rights

(c) 2015 IEEE. Personal use of this material is permitted. Permission from IEEE must be obtained for all other uses, in any current or future media, including reprinting/republishing this material for advertising or promotional purposes, creating new collective works, for resale or redistribution to servers or lists, or reuse of any copyrighted component of this work in other works.

\section{General rights}

Copyright for the publications made accessible via the Queen's University Belfast Research Portal is retained by the author(s) and / or other copyright owners and it is a condition of accessing these publications that users recognise and abide by the legal requirements associated with these rights.

Take down policy

The Research Portal is Queen's institutional repository that provides access to Queen's research output. Every effort has been made to ensure that content in the Research Portal does not infringe any person's rights, or applicable UK laws. If you discover content in the Research Portal that you believe breaches copyright or violates any law, please contact openaccess@qub.ac.uk. 


\section{Improving Signal Reliability in Outdoor Body-to- Body Communications Using Front and Back Positioned Antenna Diversity}

\author{
Simon L. Cotton ${ }^{1}$, William G. Scanlon ${ }^{2}$ \\ Institute of Electronics, Communications \& IT (ECIT) \\ Queen's University Belfast \\ Belfast, United Kingdom \\ \{simon.cotton ${ }^{1}$,w.scanlon $\left.{ }^{2}\right\} @$ qub.ac.uk
}

\author{
Adrian McKernan \\ ACT Wireless Ltd. \\ Institute of Electronics, Communications \& IT (ECIT) \\ Belfast, United Kingdom \\ adrian@act-wireless.com
}

\begin{abstract}
It has previously been shown that human body shadowing can have a considerable impact on body-to-body communications channels in low multipath environments. Signal degradation directly attributable to shadowing when one user's body obstructs the main line of sight can be as great as $40 \mathrm{~dB}$. When both people's bodies obstruct the direct line of sight path, the communications link can be lost altogether even at very short distances of a few metres. In this paper, using front and back positioned antennas, we investigate the utility of a simple selection combination diversity combining scheme with the aim of mitigating human body shadowing in outdoor body-to-body communications channels at $2.45 \mathrm{GHz}$. Early results from this work are extremely promising, indicating substantial diversity gains, as great as $29 \mathrm{~dB}$, may be achieved in a number of everyday scenarios likely to be encountered in body-to-body networking.
\end{abstract}

Keywords- Body centric communications, body-to-body networks, spatial diversity

\section{INTRODUCTION}

Body-to-body communications have recently become the focus of much research interest due to the desire to share realtime information between co-located people [1, 2]. When operated in this mode, wireless devices, either carried or worn by people can be used to form a new type of network known as a body-to-body network (BBN). When operating in a cooperative manner, BBNs will offer many attractive benefits to society including the ability to carry voice, video and data communications to extend the range of mobile networks as well as creating a range of new social networking applications.

As body-to-body communications occur between wireless devices situated on different human bodies, signal propagation will be particularly susceptible to human body shadowing [3, 4]. Human body shadowing occurs when a person's body obstructs the main line of sight (LOS) signal path between the transmitter and receiver. In a previous study by the authors [3] it has been shown that this shadowing factor may be as great as $40 \mathrm{~dB}$. Moreover, this situation may be further exacerbated when both people's bodies are orientated such that they obstruct the main LOS path, creating a so-called dual-body shadowing event, which can cause the body-to-body communications link to be lost altogether, even at a modest separation distance of a few metres [3]. An undesired side effect of these channel conditions will be the potential for extended periods of outage especially when BBNs are operating in low multipath surroundings such as outdoor environments. In this type of situation, spatial diversity based upon front and back positioned antennas may help to improve signal reliability and hence overall system performance. In this paper, using front and back positioned antennas operating at $2.45 \mathrm{GHz}$, we will investigate the utility of some of the most commonly encountered diversity combining techniques with the aim of mitigating human body shadowing in outdoor bodyto-body communications channels.

\section{EXPERIMENTAL SET-UP AND MEASUREMENTS}

\section{A. Hardware}

The bodyworn nodes used in this study consisted of the body sensor node (BSN) platform developed by Imperial College London [3]. The transceiver section of the node utilised a Texas Instruments CC2420, which has a linear dynamic operating range of approximately $100 \mathrm{~dB}$, maximum transmit power of $0 \mathrm{dBm}$ and a receive sensitivity of $-95 \mathrm{dBm}$. The BSN nodes were modified to replace the on-board chip antenna with a novel, flexible patch antenna (Fig. 1 in [3]) that was designed to be resonant on the body with a peak gain in the off-body direction of $+6.2 \mathrm{dBi}$.

\section{B. Body-to-Body Network}

To investigate the potential improvement in system performance when using spatial diversity in body-to-body communications, a BBN was formed between a node positioned on the front central chest region of an adult male (person A, node A1) and two nodes positioned on another adult male (person B). The nodes located on person B where positioned on his front central chest (node B1 or equivalently branch 1) and posterior chest (node B2 / branch 2) regions at a similar height above ground level. The nodes positioned on person $\mathrm{B}$ were then programmed to transmit packets to the node positioned on person A using a carrier sense multiple access with collision avoidance (CSMA/CA) approach to channel access. Thus when a node wishes to transmit, it performs a clear channel assessment (CCA) before 
transmitting. Upon sensing a clear channel, the node takes the opportunity and transmits. If node A1 successfully receives the transmission, it records the 8-bit received signal strength indicator (RSSI) available as a field appended to the packet demodulated by CC2420. As discussed below, this channel access scheme ensured that the median separation time between successive packets transmitted by any particular node was approximately $20 \mathrm{~ms}$.

\section{Measurements}

The measurements conducted in this study were performed in an outdoor car park adjacent to the Institute of Electronics, Communications \& IT in the Titanic Quarter of Belfast in the United Kingdom. As shown in Fig.1 (and discussed in more detail later), four individual scenarios were considered, representative of typical body-to-body networking scenarios likely to be encountered in everyday life. These were: (1) person $\mathrm{B}$ walking towards and then away from person $\mathrm{A}$ in a straight line; (2) person B walking in a line perpendicular to the direction person $\mathrm{A}$ is facing; (3) person $\mathrm{B}$ walking randomly in front of person $\mathrm{A}$; (4) person $\mathrm{B}$ walking around the front of person $\mathrm{A}$ in a semicircle from the right to left.

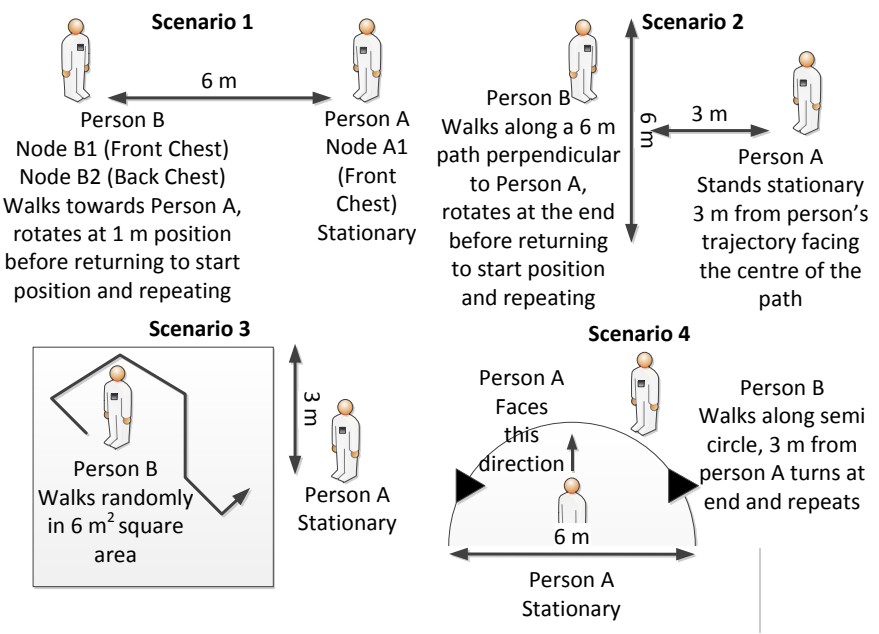

Fig. 1 Measurement scenarios.

\section{Data Analysis}

In this paper, selection combination (SC) was used to combine the signal waveforms for each of the links (node A1 / node B1 and node A1 / node B2) in post processing. Selection combination is a form of switched diversity that provides an output by selecting the branch having the highest input SNR, although in practice the branch with the largest signal plus noise contribution is used due to the difficulty in measuring SNR. Thus for a diversity array consisting of 2 branches, the SC output level $R$ is

$$
R=\max \left(r_{1}, r_{2}\right)
$$

where $r_{1}$ and $r_{2}$ are the signal levels observed in branch 1 and 2 of the diversity receiver respectively.

To simplify the analysis presented here, we assume reciprocity of the transmitter and receiver for any given link. For the experiments presented in this paper, it is anticipated due to the similar positioning of the antennas upon each person's body that channel characteristics as viewed from either end of the wireless link should be similar. This is in contrast to wireless channels commonly encountered in other communications scenarios such as cellular networks where the antenna at one end of the link (base station) may be elevated and relatively free from shadowing and multipath generated by nearby objects. Whereas, the antenna on the mobile device is at a much lower level and subject to these influences. Here the transfer characteristics of the channel as governed by reciprocity theorem are the same, but the channel characteristics which generate the transfer function not necessarily so. These may vary significantly when viewed from either the transmitter or receiver.

To create the signal waveform as seen at the output of a hypothetical bodyworn SC receiver, we down-sample the RSSI time series for each link taking synchronous samples at $100 \mathrm{~ms}$ intervals. This figure was chosen as it is well above the median packet separation time $(20 \mathrm{~ms})$ for consecutive packets for each link arriving at node A1. For example, Fig. 2 shows the cumulative distribution function (CDF) of packet separation times for both links during scenario 1 . This was representative of all other scenarios, with $90 \%$ of all consecutive packet separation times being less $30 \mathrm{~ms}$.

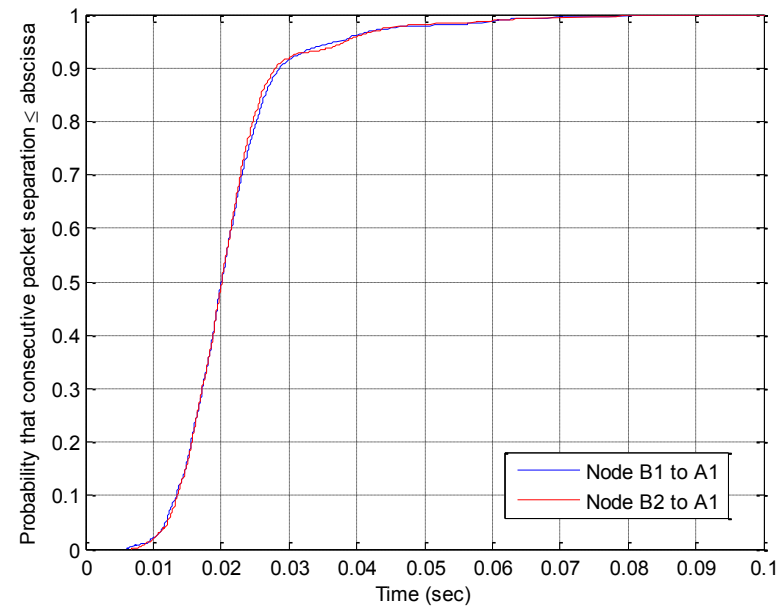

Fig. $2 \mathrm{CDF}$ of consecutive packet arrival separation times for CSMA/CA approach to channel access for each link in scenario 1 .

\section{RESULTS}

\section{A. Scenario 1}

In scenario 1 , persons $\mathrm{A}$ and $\mathrm{B}$ initially stood stationary with a separation distance of $6 \mathrm{~m}$, such that the nodes positioned on their respective front chests (A1 and B1) were in direct LOS. Data logging then commenced as person B walked towards person A with an average straight line velocity of $0.7 \mathrm{~ms}^{-1}$. When person $\mathrm{B}$ reached the point $1 \mathrm{~m}$ away from person $\mathrm{A}$, he performed a 180 degree rotation and walked back 
towards the starting point $6 \mathrm{~m}$ away, such that the node positioned on his front chest (B1) was now shadowed by his body and the node positioned on his back chest region (B2) was in LOS. This procedure was repeated three times during this scenario. A continuous segment of the RSSI time series from each link lasting approximately 35 seconds was used for data analysis. A sample set of this size was also used for all other scenarios.

Fig. 3 shows the measured (raw) received power time series for both links and the down-sampled diversity combined received power time series for scenario 1 . It can be seen quite clearly from Fig. 3, as person B begins to move towards person A, the mean signal received by branch 2 of the virtual diversity receiver does not change significantly up until the $1 \mathrm{~m}$ separation point whereas for branch 1 the mean signal level increases significantly ( $20 \mathrm{~dB}$ over the same range). Closer inspection of the received power time series for this stage of scenario 1 shows that very little short term fading is experienced by branch 1 . Conversely, branch 2 experiences quite significant signal variation over the same period, with the true depths of the short-term fades most likely truncated by the noise threshold of the receiver. This suggests that the link between branch 2 and the node on person $\mathrm{A}$ is dependent on constantly changing signal paths or path lengths possibly caused by movements of the limbs.

From Fig. 3, it is also quite clear that for this scenario, using a diversity combiner together with the front and back positioned antennas, a significant improvement in signal reliability could be achieved. This can be seen at the $1 \mathrm{~m}$ points annotated on Fig. 3. For example, looking at the first $1 \mathrm{~m}$ point, when person $\mathrm{B}$ begins to rotate, the link between the node A1 and B1 on person B's front chest begins to enter a shadowing region. As this occurs, the link between A1 and B2 immediately begins to improve sustaining the link well above the noise threshold of the system. A figure which can be used to quantify the improvement in signal reliability of a diversity receiver over a single branch system is diversity gain. It is defined as the difference in the signal level of the branch with the highest mean and that of the output of the diversity combiner for a given probability or signal reliability (in this case $90 \%$ or a cumulative probability of 0.1 ). As shown in Fig. 4, using the front and back positioned antennas and a selection combination combining scheme for scenario 1 , a quite considerable diversity gain of $29 \mathrm{~dB}$ was obtained compared to using one signal branch in isolation.

Another interesting observation made from Fig. 3 is that, when using the relatively directive flexible patch antennas considered in this study, the impact of human body shadowing appears to increase with a decreasing separation distance between the transmitter and receiver. This can be seen by monitoring the difference in signal level between branches 1 and 2 between the $6 \mathrm{~m}$ and $1 \mathrm{~m}$ separation points. Due to body shadowing this difference increases from around $30 \mathrm{~dB}$ up to $40 \mathrm{~dB}$ showing that at $2.45 \mathrm{GHz}$, the human body is a significant shadowing object. Furthermore, this drop in received signal power may be detrimental for single antenna receivers used in body-to-body links which extend beyond the distances considered here.

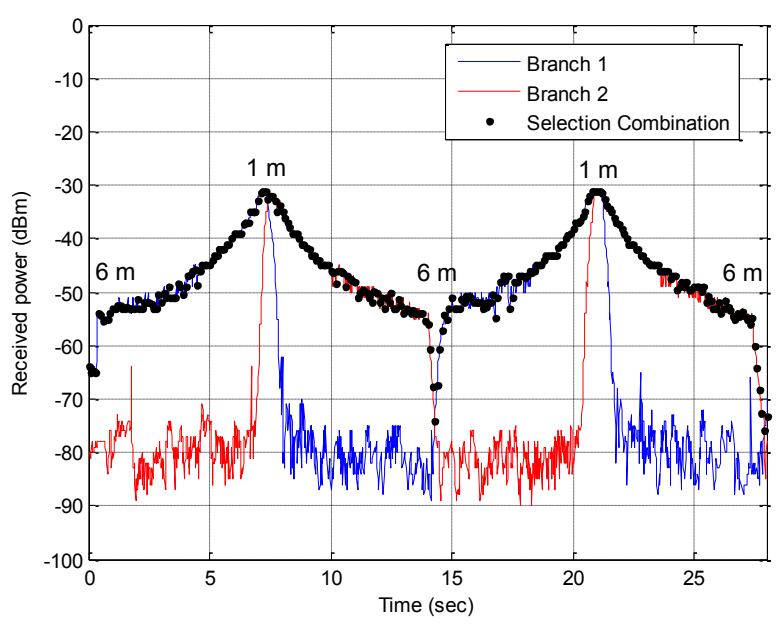

Fig. 3 Received power time series for input branches 1 and 2 of the diversity receiver alongside the hypothetical selection combination output during scenario 1 .

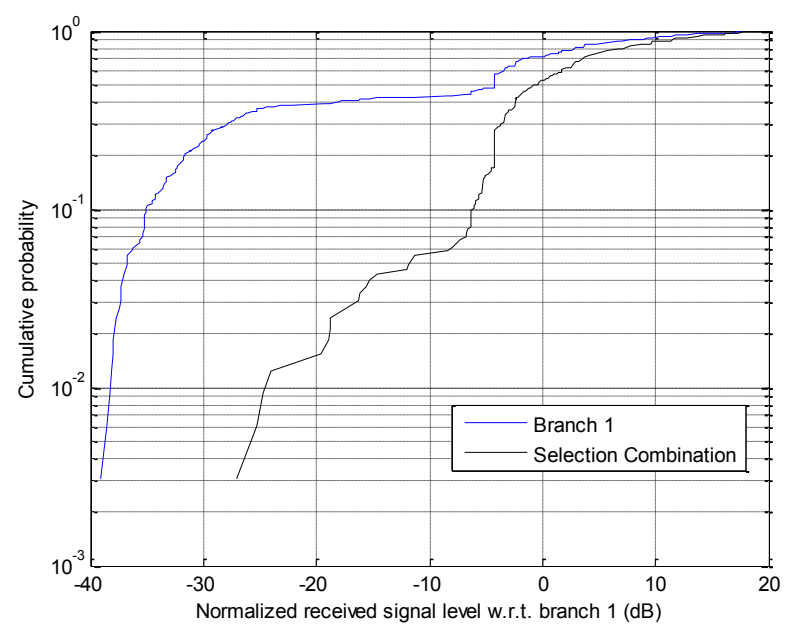

Fig. 4 Empirical cumulative distribution functions for branch 1 (highest mean) and the output of a hypothetical selection combination combing scheme using front and back positioned antennas for scenario 1 .

\section{B. Scenario 2}

In scenario 2 (Fig. 1), person B walked along a $6 \mathrm{~m}$ line while person A stood stationary facing perpendicular to the centre point of the line at a distance of $3 \mathrm{~m}$. Upon reaching the end point of the line, person B turned and walked back to his starting position, this process was then repeated a further two times (three in total). For scenario 2, because both antennas situated on person B crossed in and out of LOS with node A1 much more frequently, the rate of signal variation in each of the links was seen to increase compared to scenario 1 (Fig. 5).

When person B passed the centre point of his trajectory, even though his body now begins to shadow the main LOS path between nodes $\mathrm{A} 1$ and $\mathrm{B} 1$, the drop off in the received signal power experienced by this link is not as rapid as that observed when the user was rotating in scenario 1 . The benefits of having more than one spatially separated antenna 
covering the front and back of the body are again evident from Fig. 5 where the all signal drops below $-80 \mathrm{dBm}$ are removed, although the diversity gain obtained by using an $\mathrm{SC}$ receiver for this scenario was lower than scenario 1 at $14.1 \mathrm{~dB}$.

\section{Scenarios 3 and 4}

Scenario 3 was conducted for 70 seconds of which approximately 35 seconds of continuous data were used for analysis. As shown in Fig. 1 it involved person B walking in a random manner, which included walking towards, away and rotating within a $6 \mathrm{~m}^{2}$ square area directly in front of person A, who stood stationary at the $3 \mathrm{~m}$ point along one side of the square. By employing a $\mathrm{SC}$ receiver the received signal power was maintained above $-70 \mathrm{dBm}$ throughout this scenario (Fig. 6). The overall diversity gain, $21.7 \mathrm{~dB}$, was also improved compared to that obtained for scenario 2 .

In scenario 4, person B walked in a semicircle from a point $3 \mathrm{~m}$ to the right of person $\mathrm{A}$ through a point $3 \mathrm{~m}$ directly in front of person $\mathrm{A}$ to a position $3 \mathrm{~m}$ to the left of person $\mathrm{A}$ before returning to his starting position (Fig. 1). This process was repeated three times in total. Of all the scenarios considered in this study, scenario 4 offered the lowest diversity gain of $1.9 \mathrm{~dB}$ (Fig. 7). There were a number possible of explanations for this observation. Firstly due to the geometry of this scenario (Fig. 1), neither node positioned on person $\mathrm{B}$ was fully shadowed by the human body (with the exception of a brief rotation at either end point of the walk path). Secondly (not shown), branch 1 of the diversity combiner (link A1 to B1) experienced a higher signal level for the majority of the experiment.

\section{CONCLUSIONS}

As shown in this paper, implementing spatial diversity for outdoor body-to-body communications systems can be expected to improve signal reliability, especially when one antenna becomes shadowed by the human body. Using a simple selection combination combing scheme with front and back positioned antennas, significant diversity gain can be achieved in many everyday scenarios. This will help to mitigate the negative effects of human body shadowing on BBN links.

\section{REFERENCES}

[1] S. L. Cotton \& W. G. Scanlon, "Using smart people to form future mobile wireless networks," Microwave Journal, vol. 54, no. 12, pp. 24 40, Dec. 2011.

[2] W. Yu, I. B. Bonev, J. O. Nielsen, I. Z. Kovacs, G. F. Pedersen, "Characterization of the Indoor Multiantenna Body-to-Body Radio Channel," IEEE Transactions on Antennas and Propagation, vol. 57, no. 4, pp. 972-979, April 2009.

[3] S. L. Cotton, A. McKernan \& W. G. Scanlon, "Received signal characteristics of outdoor body-to-body communications channels at 2.45 GHz," Loughborough Antennas \& Propagation Conference, Nov. 2011.

[4] S. Obayashi and J. Zander, "A Body-Shadowing Model for Indoor Radio Communication Environments", IEEE Trans on Antennas and Prop., vol.46, no. 6, pp. 920-927, June 1998.

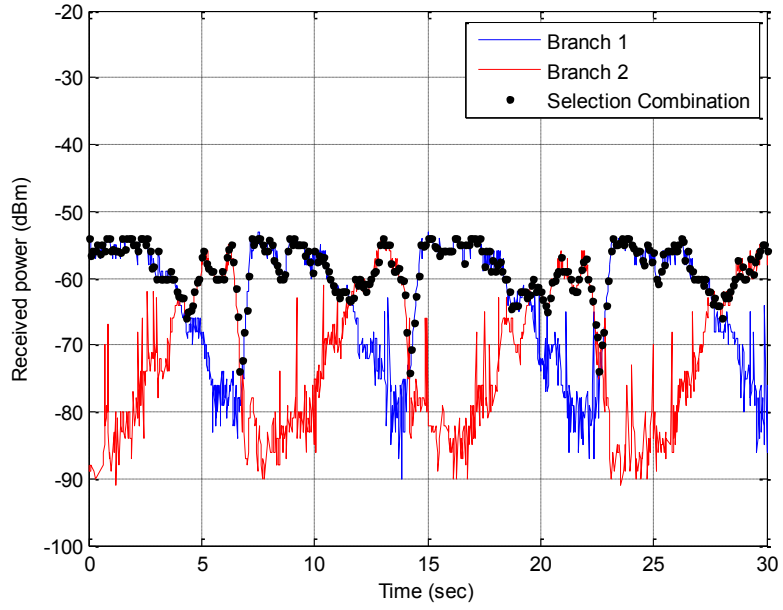

Fig. 5 Received power time series for input branches 1 and 2 of the diversity receiver alongside the hypothetical selection combination output during scenario 2 .

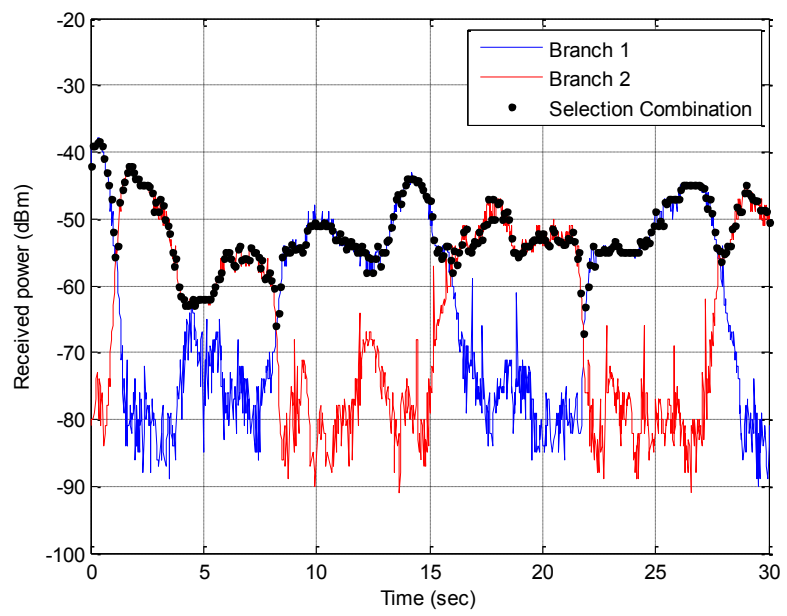

Fig. 6 Received power time series for input branches 1 and 2 of the diversity receiver alongside the hypothetical selection combination output during scenario 3 .

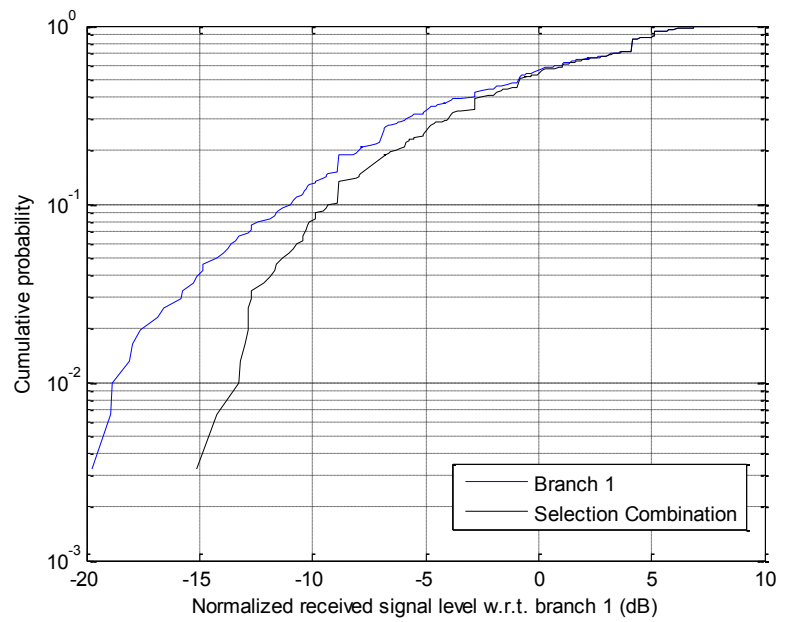

Fig. 7 Empirical cumulative distribution functions for branch 1 (highest mean) and the output of a hypothetical selection combination combing scheme using front and back positioned antennas for scenario 4 . 